\title{
Theoretical development of minimum effort active noise control with feedback inclusion architecture.
}

\begin{abstract}
This paper presents the development of minimum effort active noise control theory for feedforward single-input single output (SISO) architecture, which includes the feedback acoustic path in the controller formulation. The theoretical range of effective minimum effort parameter with respect to level of point cancellation at the observer and the interference pattern generated around the observer for periodic noise control in free-field are investigated. It is found that for a minimum of $6 \mathrm{~dB}$ cancellation which corresponds to cancellation factor of 0.75 at the observer, the effort parameter must be specified as less than unity. It is also found that the cancellation pattern characterised by $20 \mathrm{~dB}, 10 \mathrm{~dB}$ and $6 \mathrm{~dB}$ zones reduces significantly in terms of size with an increase in the value of the effort term.
\end{abstract}

Keyword: Noise control; Feed-forward; Single-input single output. 